\title{
Shielding Effect of Nanomicelles: Stable and Catalytically Active Oxidizable Pd(0) Nanoparticle Catalyst Compatible for Cross- Couplings of Water-Sensitive Acid Chlorides in Water
}

Tharique N. Ansari, Sudripet Sharma, Susanta Hazra, Jacek B. Jasinski, Andrew J. Wilson, Frederick Hicks, David K. Leahy,* and Sachin Handa*

Cite This: JACS Au 2021, 1, 1506-1513

Read Online

ABSTRACT: Under the shielding effect of nanomicelles, a sustainable micellar technology for the design and convenient synthesis of ligand-free oxidizable ultrasmall $\mathrm{Pd}(0)$ nanoparticles (NPs) and their subsequent catalytic exploration for couplings of water-sensitive acid chlorides in water is reported. A prolinederived amphiphile, PS-750-M, plays a crucial role in stabilizing these NPs, preventing their aggregation and oxidation state changes. These NPs were characterized using ${ }^{13} \mathrm{C}$ nuclear magnetic resonance (NMR), infrared (IR), and surface-enhanced Raman scattering (SERS) spectroscopy to evaluate the carbonyl interactions of PS-750-M with Pd. High-resolution transmission electron microscopy (HRTEM) and energy-dispersive X-ray

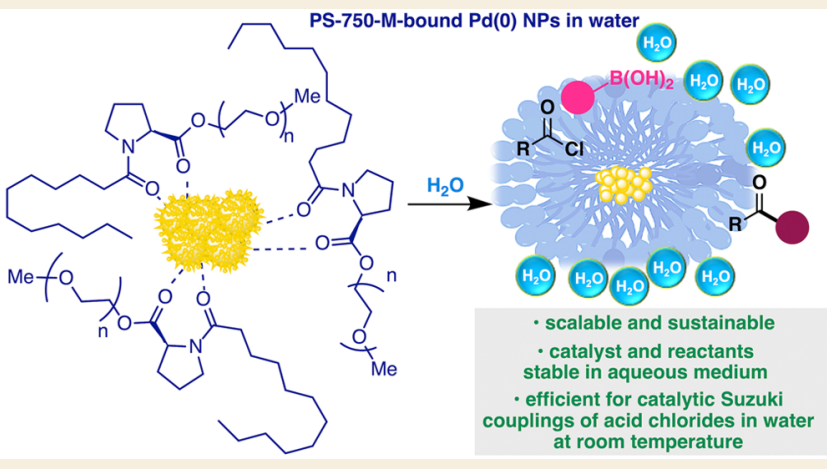
spectroscopy (EDX) studies were performed to reveal the morphology, particle size distribution, and chemical composition, whereas X-ray photoelectron spectroscopy (XPS) measurements unveiled the oxidation state of the metal. In the cross-couplings of water-sensitive acid chlorides with boronic acids, the micelle's shielding effect and boronic acids plays a vital role in preventing unwanted side reactions, including the hydrolysis of acid chlorides under basic $\mathrm{pH}$. This approach is scalable and the applications are showcased in multigram scale reactions.

KEYWORDS: micellar catalysis, chemistry in water, acid chloride, nanoparticle, sustainability

\section{INTRODUCTION}

Palladium (Pd) serves as the preferred metal for catalysis in modern organic synthesis, ${ }^{1-3}$ as evidenced by its applications in several industrial processes ${ }^{4-6}$ and the 2010 Nobel Prize in chemistry. ${ }^{1,7,8}$ Its exceptional catalytic activity in constructing bonds between highly functionalized molecules stems from its ability to readily switch between oxidation states for achieving the desired reaction pathway. ${ }^{9,10}$ In general, most Pd-mediated catalytic processes operate under $\mathrm{Pd}(0)$ to $\mathrm{Pd}(\mathrm{II})$ or $\mathrm{Pd}(\mathrm{II})$ to $\mathrm{Pd}(\mathrm{IV})$ cycles. ${ }^{11}$ The latter two-electron catalytic cycle's scope and sustainability are often limited by the requirement of stronger oxidants and/or directing groups in the substrate, ${ }^{12,13}$ whereas the $\mathrm{Pd}(0) / \mathrm{Pd}(\mathrm{II})$ cycle can be attained using a broad range of electrophiles and nucleophiles under mild conditions. ${ }^{2,14}$ Many significant advancements have been reported demonstrating the efficient carbon-carbon and carbonheteroatom bond construction, ${ }^{2,14-16}$ often relying on new catalyst designs to generate and stabilize the catalytically essential $\operatorname{Pd}(0)$ species. These generally focus on fine-tuning the steric and electronic parameters associated with the ligand bound to the Pd atom. ${ }^{16-18}$ The morphology of the catalyst, especially in heterogeneous and quasi-homogeneous forms, also plays a critical role in catalyst efficiency.

Recently, nanocatalysis (quasi-homogeneous) has emerged as a powerful tool to enable cross-couplings under mild conditions. $^{19-22}$ Because of their improved surface to volume ratio, nanoparticle (NP) catalysts exhibit enhanced activity, $^{20,21,23,24}$ selectivity, ${ }^{25,26}$ and recyclability ${ }^{20,21,27}$ compared to their homogeneous organometallic counterparts. These properties rely significantly on the NP's morphology, size, and oxidation state. The nature of the ligand/capping agent and the kind of nucleation determine the size, shape, and morphology of resulting NPs. ${ }^{28-30}$ Generally, phosphines or nitrogen-based ligands are required for the desired properties. ${ }^{20,21,27}$ Often, such ligands adversely affect the sustainability parameters. $^{20,21,27,31}$ In the absence of such ligands, $\operatorname{Pd}(0)$ readily

Received: May 27, 2021

Published: July 20, 2021 


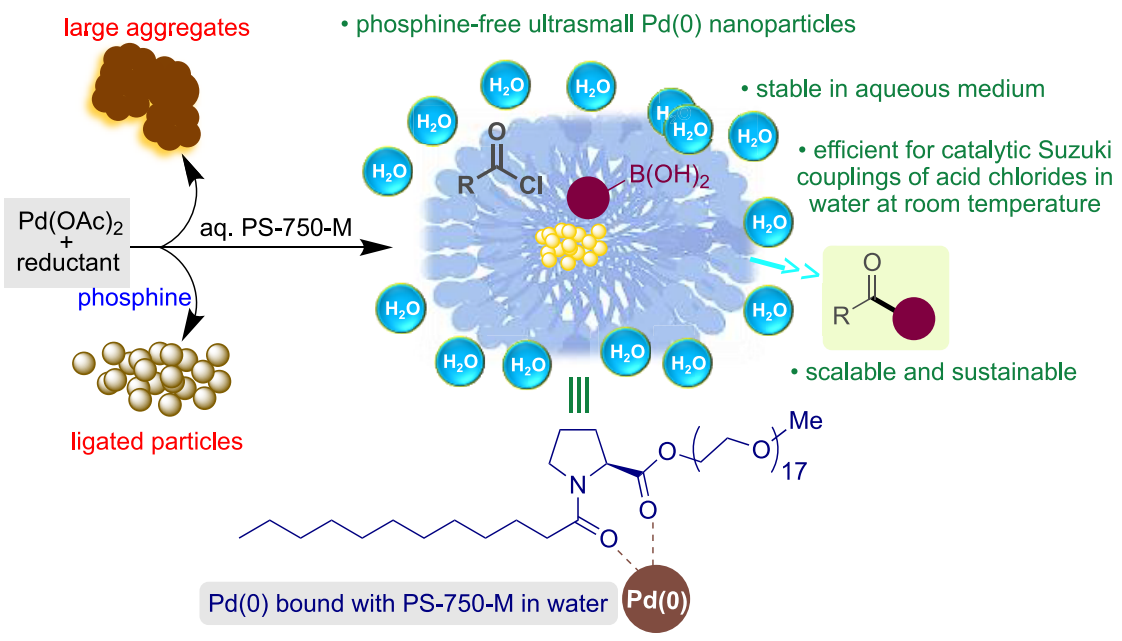

Figure 1. Phosphine ligand-free $\mathrm{Pd}(0)$ NPs for sustainable $\mathrm{C}-\mathrm{C}$ couplings.

oxidizes to Pd(II), causing agglomeration of NPs. Removing these ligands without compromising the catalytic activity and aforementioned physical nature of NPs is always highly desirable, especially for large-scale applications where cost, sustainability, and ease of product purification are critical. Hence, solvent-stabilized $\mathrm{Pd}(0)$ NPs have recently emerged as a viable alternative. ${ }^{32-34}$ Traditionally, the synthesis of such NPs relies on toxic organic solvents, such as $\mathrm{DMF}^{34,35}$ and $\mathrm{NMP},{ }^{36}$ where the solvent's tertiary amide group plays a vital role in stabilizing the NP.

Designing an amphiphile possessing structural features similar to DMF and NMP could enable the synthesis, stabilization, and applications of phosphine-free $\operatorname{Pd}(0)$ NPs under aqueous conditions. This synthetic approach could be of interest from a sustainability perspective as water serves as the green and preferred solvent. ${ }^{37-39}$ Realizing the aforementioned needs while advancing chemistry in water, our laboratory has developed a proline-based amphiphile PS-750-M that mimics dipolar-aprotic solvents, such as DMF, NMP, and DMAc. ${ }^{40-44}$ Incorporating a tertiary amide group into the amphiphile helps stabilize the NPs and facilitate their delivery into the micellar interior or polar-nonpolar interface. ${ }^{45-47}$ Our group recently leveraged this amphiphile's ability to stabilize Pd(II) NPs and employed it in efficient oxidative Mizoroki-Heck type couplings. ${ }^{48}$ Stabilization of Pd(II) NPs and their accommodation in the micellar interface enabled oxidative cross-couplings under mild conditions. Herein, we report a phosphine ligand-free synthesis of ultrasmall $\operatorname{Pd}(0)$ NPs from Pd(II) salt in an aqueous micellar solution and their applications in cross-couplings of water-sensitive acid chlorides (Figure 1). We likewise demonstrate their utility in traditional Suzuki-Miyaura couplings, which are established workhorses of organic synthesis. The broad utility of these catalysts are a consequence of uniformly distributed ultrasmall NPs stabilized by PS-750-M.

\section{RESULTS AND DISCUSSION}

Our study began with the synthesis of $\operatorname{Pd}(0)$ NPs, achieved by suspending $\mathrm{Pd}(\mathrm{OAc})_{2}$ as the $\mathrm{NP}$ precursor in an aqueous solution of PS-750-M and reducing it with phenylboronic acid under basic $\mathrm{pH}$. The base activates the phenylboronic acid, causing transmetalation on $\mathrm{Pd}(\mathrm{II})$ by phenyl nucleophiles. The fast reductive elimination of biphenyl, rapid nucleation, and in situ capping of resulting NPs with PS-750-M provide ultrasmall and highly active $\operatorname{Pd}(0)$ NPs (Scheme 1; for details, see page S2 in the Supporting Information). This mild synthetic route is

Scheme 1. Nanoparticle Synthesis

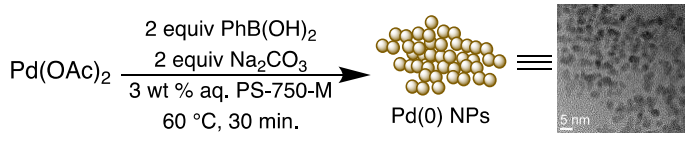

scalable and completely avoids harsh reductants $(\mathrm{MeMgBr}$, $\mathrm{LAH}$, or $\left.\mathrm{NaBH}_{4}\right)$ and toxic organic solvents $\left(\mathrm{CH}_{2} \mathrm{Cl}_{2}\right.$, THF). ${ }^{20,21,47,49}$ The NPs obtained by this mild technology were characterized using a variety of analytical techniques. Infrared (IR) and ${ }^{13} \mathrm{C}$ nuclear magnetic resonance (NMR) spectroscopy, and surface-enhanced Raman scattering (SERS) were used for probing the interaction between $\mathrm{Pd}$ and the carbonyls of PS-750-M, whereas high-resolution transmission electron microscopy (HRTEM) was employed to examine morphology and particle size distribution. Energy-dispersive Xray spectroscopy (EDX) mapping allows for the determination of the chemical composition, and X-ray photoelectron spectroscopy (XPS) allows for studying the metal's oxidation state.

${ }^{13} \mathrm{C}$ NMR studies were performed to probe the interaction of the carbonyl functional groups of PS-750-M with Pd (Figure 2A; also see, page S6 of the Supporting Information). The ester and amide carbonyls of the free amphiphile appeared at 173.4 and $173.1 \mathrm{ppm}$, respectively, whereas the acetate carbonyl of $\mathrm{Pd}(\mathrm{OAc})_{2}$ appeared at $172.8 \mathrm{ppm}$. The multiplicity of ${ }^{13} \mathrm{C}$ signals from the carbonyls of PS-750-M are due to the presence of $\mathrm{mPEG}$ of different chain lengths having an average molecular weight of $750 \mathrm{Da}$ and the presence of variable-sized micelles. The ${ }^{13} \mathrm{C}$ NMR spectrum of the NPs was significantly different compared to that of $\mathrm{Pd}(\mathrm{OAc})_{2}$ and PS-750-M. Multiple signals at 175.5-173.8 and 183.4-180.3 ppm indicate the amphiphile's carbonyls binding with $\mathrm{Pd}$. The signal's complex multiplicity is most likely due to the formation of different sized micelles. To confirm metal-amphiphile interactions, infrared spectroscopic (IR) studies were also performed (see page S5 of the Supporting Information). In the IR spectrum of the metal-free PS-750-M, the stretching frequency of carbonyl functional groups appears at 1711 and $1640 \mathrm{~cm}^{-1}$. The ester carbonyl of the $\mathrm{Pd}(\mathrm{OAc})_{2}$ shows a 

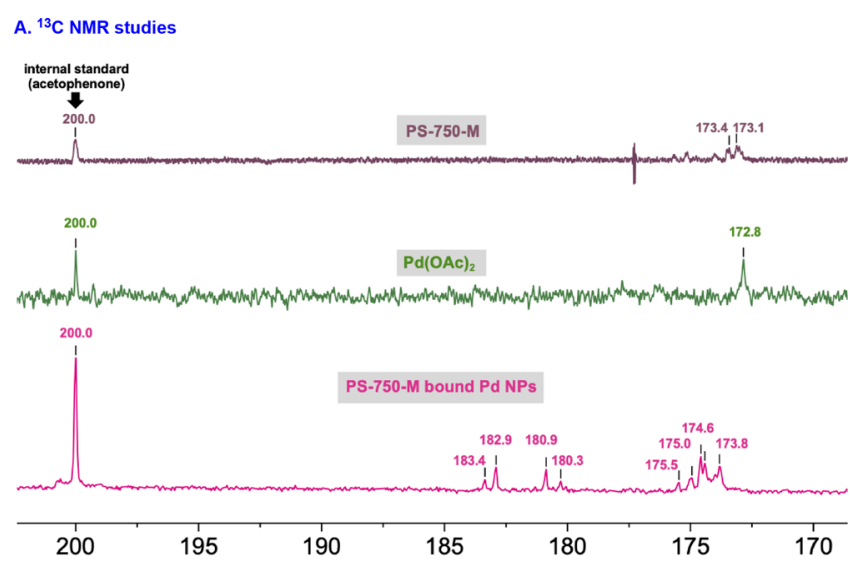

B. IR studies

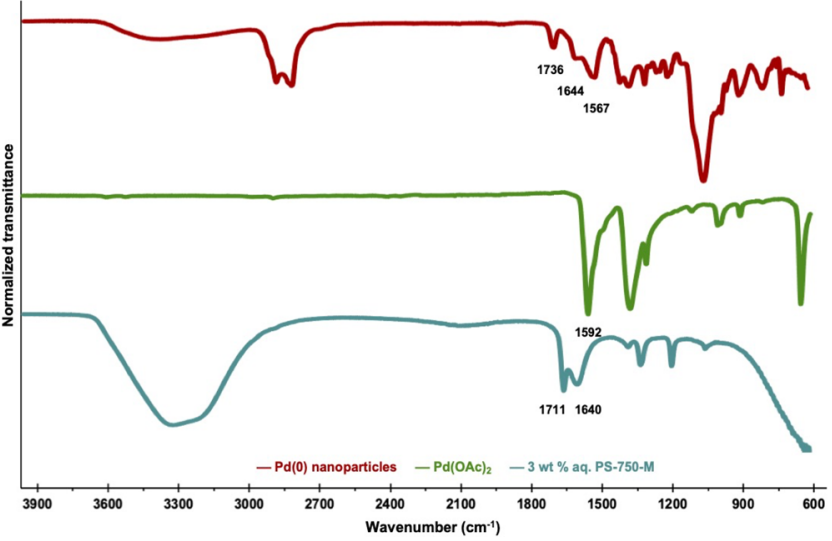

Figure 2. (A) ${ }^{13} \mathrm{C}$ NMR study of the NPs, (B) IR study of the NPs.

stretch at $1592 \mathrm{~cm}^{-1}$. However, the IR spectrum of the NPs was distinct from PS-750-M and $\mathrm{Pd}(\mathrm{OAc})_{2}$. A new set of signals appeared at 1736,1644 , and $1567 \mathrm{~cm}^{-1}$ suggesting binding of the amphiphile's carbonyls with the metal NP (Figure 2B).

To further confirm the Pd-PS-750-M interaction and probe the functional groups responsible for interaction, we performed SERS analysis of PS-750-M-bound Pd NPs, neat PS-750-M, and $\mathrm{Pd}(\mathrm{OAc})_{2}$. Figure 3 shows a SERS spectrum of Pd NPs encapsulated by micelles of PS-750-M (red curve). Vibrational modes with peaks at 1615 and $1762 \mathrm{~cm}^{-1}$ are assigned to the carbonyl vibrations from the amide and ester, respectively. The SERS spectrum from neat PS-750-M without Pd (blue curve) shows an amide carbonyl vibration at $1629 \mathrm{~cm}^{-1}$ and an ester carbonyl vibration at $1771 \mathrm{~cm}^{-1}$. The decrease in Raman shift of the carbonyl vibrational modes with Pd present indicates binding of the carbonyls with the $\mathrm{Pd}$, which weakens the $\mathrm{C}=$ $\mathrm{O}$ bond and causes the shift in peak frequency. Peaks at 1475, 1316 , and $987 \mathrm{~cm}^{-1}$ in the neat PS-750-M also shift to 1423 , 1311, and $949 \mathrm{~cm}^{-1}$ when Pd NPs are present. These vibrational modes are likely associated with the carbonyl carbon and amide/ester nitrogen/oxygen atoms (e.g., OCN, $\mathrm{CCN}, \mathrm{OCO}, \mathrm{CCO}$ ) supporting the assertion that the carbonyl is physisorbed to Pd. A Raman spectrum from a precursor $\mathrm{Pd}(\mathrm{OAc})_{2}$ crystal is shown for comparison (black curve). Therefore, based on SERS, IR, and NMR analysis, the ester and amide functional groups of PS-750-M are responsible for metal-micelle binding.

HRTEM analysis indicated the formation of ultrasmall $\mathrm{Pd}$ NPs with an average size of $2.41 \mathrm{~nm}$ (Figure 4A-C). Likewise,

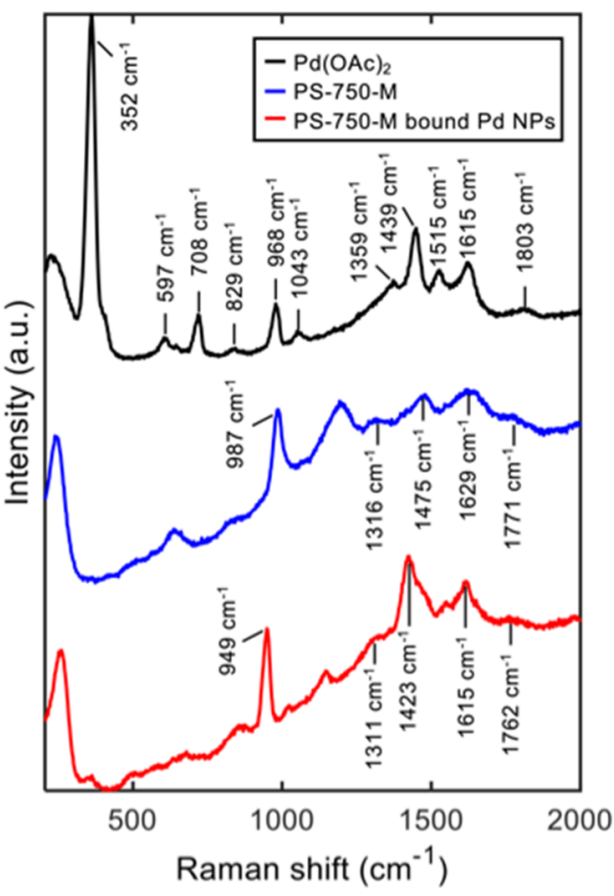

Figure 3. Surface-enhanced Raman scattering (SERS) spectra of the catalyst and its components. Raman spectrum of a $\mathrm{Pd}(\mathrm{OAc})_{2}$ crystal (black), SERS spectrum of PS-750-M without $\mathrm{Pd}(0)$ NPs (blue), and SERS spectrum from Pd(0) NPs encapsulated by PS-750-M (red).

scanning transmission electron microscopy-high-angle annular dark-field imaging (STEM-HAADF) reveals the NPs formation. However, these particles tend to aggregate easily under high-energy electron beam exposure limiting the acquisition of STEM-HAADF imaging (Figure 4D; see pages S3 and S4 in the Supporting Information). EDX mapping reveals the presence of the nitrogen from the amphiphile and Pd in the NPs (Figure 4E). These combined techniques confirmed the formation of ultrasmall Pd NPs. Further, the XPS analysis of the anhydrous NPs confirms the presence of both the $\mathrm{Pd}(\mathrm{II})$ and $\mathrm{Pd}(0)$ based on their distinct binding energies in the peak deconvolution spectrum of $\mathrm{Pd}$ $3 \mathrm{~d} 5 / 2$ (Figure 4F; see page S7 in the Supporting Information). Most likely, the presence of $\mathrm{Pd}(\mathrm{II})$ arises from the aerobic oxidation of surface $\mathrm{Pd}(0)$ to $\mathrm{Pd}(\mathrm{II})$ during the drying process.

Because of the facile transmetalation of arylboronic acids to PS-750-M-stabilized Pd NPs, our evaluation on the catalytic activity of these $\operatorname{Pd}(0)$ NPs started with the cross-couplings of acid chlorides and boronic acids, providing access to functionalized ketones under mild aqueous conditions. ${ }^{50-52}$ Recognizing the widespread distribution of these compounds in pharmaceuticals, agrochemicals, and fine chemicals, ${ }^{53-55}$ this transformation could be a greener alternative compared to the traditional multistep route involving Grignard addition to an aldehyde followed by oxidation of the resulting alcohol. ${ }^{56,57}$ Therefore, we investigated the possibility of such a $\mathrm{C}-\mathrm{C}$ coupling reaction catalyzed by phosphine-free $\operatorname{Pd}(0)$ NPs using benzoyl chloride (1) and 4-(trifluoromethyl)phenylboronic acid (2) as benchmark substrates under aqueous micellar conditions.

Optimization studies revealed the dependence of the choice of the reaction medium, catalyst, base, and global concentration on the reaction outcome (Table 1; see pages S10-S12 in the Supporting Information). Optimal conditions involve 3 

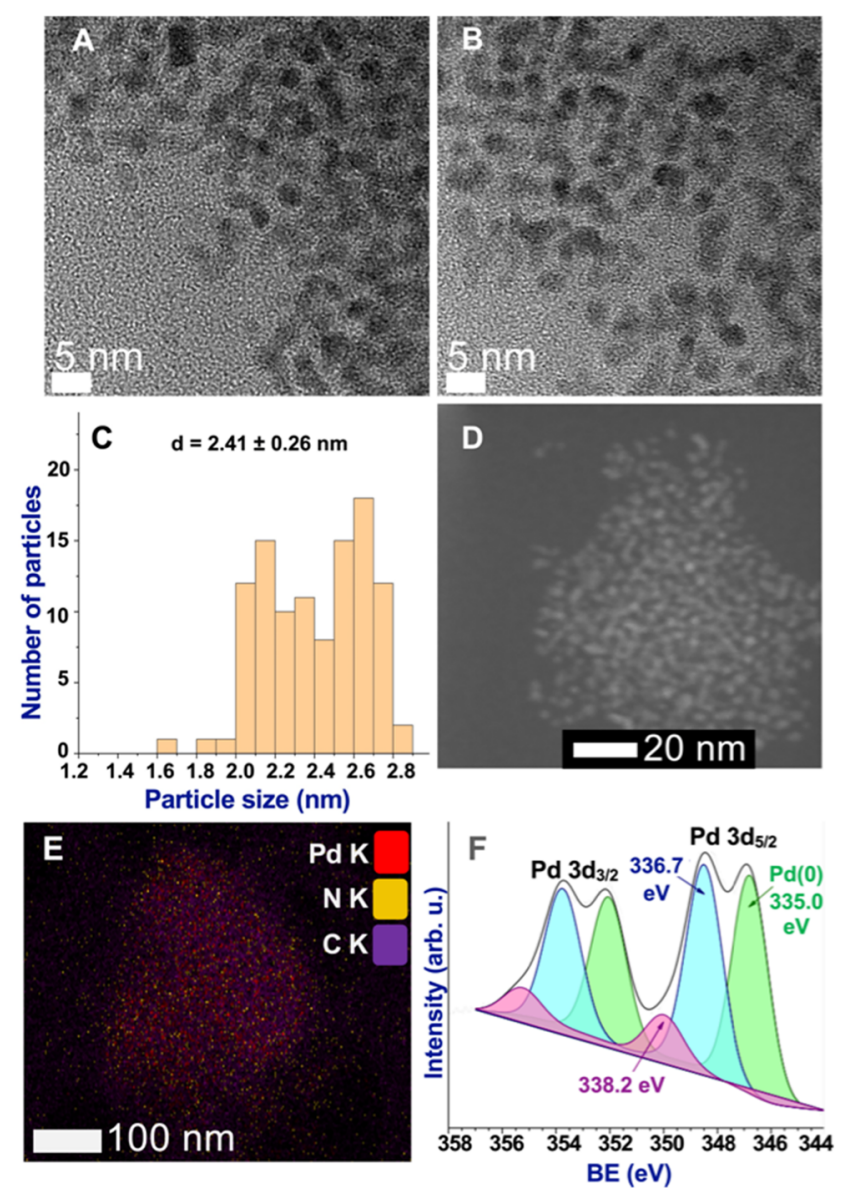

Figure 4. (A-C) HRTEM images and histogram of the NPs. (D) STEM-HAADF image. (E) EDX mapping analysis. (F) Peak deconvoluted XPS spectra of Pd.

Table 1. Optimization Studies

\begin{tabular}{|c|c|c|c|}
\hline & 2 & $\begin{array}{c}3 \mathrm{~mol} \% \mathrm{Pd}(0) \mathrm{NPs} \\
2.0 \text { equiv } \mathrm{Na}_{2} \mathrm{CO}_{3} \\
3 \text { wt } \% \text { aq. PS-750-M } \\
0.25 \mathrm{M}, \mathrm{rt}, 3 \mathrm{~h}\end{array}$ & 3 \\
\hline entry & \multicolumn{2}{|c|}{ deviations from std. conditions $^{a}$} & $3(\%)^{b}$ \\
\hline 1 & \multicolumn{2}{|l|}{ none } & $94(90)^{c}$ \\
\hline 2 & \multicolumn{2}{|c|}{ Ni NPs instead of Pd } & traces \\
\hline 3 & \multicolumn{2}{|c|}{$\mathrm{Cu}$ NPs instead of Pd } & traces \\
\hline 4 & \multicolumn{2}{|c|}{$\mathrm{Et}_{3} \mathrm{~N}$ instead of $\mathrm{Na}_{2} \mathrm{CO}_{3}$} & 32 \\
\hline 5 & \multicolumn{2}{|c|}{$\mathrm{KOH}$ instead of $\mathrm{Na}_{2} \mathrm{CO}_{3}$} & $40^{d}$ \\
\hline 6 & \multicolumn{2}{|c|}{$\mathrm{H}_{2} \mathrm{O}$ instead of PS-750-M } & $53^{e}$ \\
\hline 7 & \multicolumn{2}{|c|}{ THF instead of PS-750-M } & $66^{f}$ \\
\hline 8 & \multicolumn{2}{|c|}{$0.5 \mathrm{~mol} \%$ of $\mathrm{Pd}(0) \mathrm{NPs}$} & 52 \\
\hline
\end{tabular}

${ }^{a}$ Conditions: $1(0.25 \mathrm{mmol}), 2(0.275 \mathrm{mmol}), \mathrm{NP}$ catalyst $(3 \mathrm{~mol}$ \%), $\mathrm{Na}_{2} \mathrm{CO}_{3}(0.5 \mathrm{mmol}), 1 \mathrm{~mL}$ of $3 \mathrm{wt} \%$ aq. PS-750-M, $23{ }^{\circ} \mathrm{C}, 3 \mathrm{~h}$. ${ }^{b}$ Conversions to 3 based on GC-MS using mesitylene $(0.25 \mathrm{mmol})$ as internal standard. ${ }^{c}$ Isolated yield. ${ }^{d} 27 \%$ homocoupling of 2 was observed. ${ }^{e} 8 \%$ homocoupling of 2 was observed. ${ }_{2} 1 \%$ homocoupling of 2 was observed.

mol \% $\mathrm{Pd}(0)$ NPs, 2.0 equiv. of $\mathrm{Na}_{2} \mathrm{CO}_{3}$ as base, and 3 wt \% aq. PS-750-M as reaction medium with a $0.25 \mathrm{M}$ global concentration, providing $94 \%$ conversion and $90 \%$ isolated yield of the desired product 3 (entry 1). Replacing Pd with earth-abundant metals, such as $\mathrm{Ni}$ or $\mathrm{Cu}$, results in traces of 3 (entries 2, 3). Replacing the base $\mathrm{Na}_{2} \mathrm{CO}_{3}$ with $\mathrm{Et}_{3} \mathrm{~N}$ or $\mathrm{KOH}$ drops the reaction conversion to 32 and $40 \%$, respectively (entries 4, 5). Considerable homocoupling of boronic acid 2 was observed $(27 \%)$ when $\mathrm{KOH}$ base was used. The poor catalytic activity observed may be due to the competitive binding of $\mathrm{Et}_{3} \mathrm{~N}$ with $\mathrm{Pd}$ occupying the catalytic sites. Likewise, the use of $\mathrm{KOH}$ may cause faster hydrolysis of benzoyl chloride or disintegration of Pd-amphiphile binding. The importance of the amphiphile as a reaction medium in this catalytic reaction is evident as its removal provides only $53 \%$ conversion to 3 (entry 6); most likely, the acid chloride's simultaneous reactivity with water is responsible for low conversion. Switching the reaction medium to THF is also detrimental, forming only $66 \% 3$ (entry 7) along with the homocoupling of $\mathbf{2}$ and unreacted acid chloride, indicating the importance of aqueous micellar conditions for the desired reactivity. Deviation from optimal catalyst loading to 5000 ppm ( $0.5 \mathrm{~mol} \%)$ provides $52 \%$ of the desired product in GCMS analysis (entry 8; for details on catalyst loading, see page S10 in the Supporting Information). Furthermore, increasing the temperature to $45{ }^{\circ} \mathrm{C}$ with this low catalyst loading affords only $37 \%$ GC-MS conversion to 3 along with hydrolysis of the acid chloride (for details, see page S10 in the Supporting Information). Most notably, the reaction's outcome signifcantly depends on the order of addition; the acid chloride must be added after cooling the mixture to $0{ }^{\circ} \mathrm{C}$ (for details, see pages S21 and S22 in the Supporting Information).

With the optimal reaction conditions established, the substrate scope was explored using combinations of various acid chlorides and aryl and heteroarylboronic acids (Table 2, 3-30). These studies indicated that the reaction outcome most likely does not rely on the coupling partner's steric and electronic factors. The scope study showed excellent functional group tolerance. Fluoro (4, 6, 7, 13, 17, 27-29), nitro (5), ester $(8)$, chloro $(4,8)$, and trifluoromethyl $(3,16,21,23,28)$ groups were well tolerated. Notably, in these examples, fluoro functional groups may be susceptible to the $S_{N} A r$ side reaction remained intact. Electron-deficient boronic acids with $o$ difluoro substituent showed good reactivity (6). Furthermore, arylboronic acids containing a terminal vinyl substituent cleanly reacted without undesired side reactions such as Heck-type coupling or polymerization, thus providing access to functionalized styrenes (11, 14, 20). Arylboronic acids possessing o-methyl $(13,17)$ or $o$-methoxy $(15)$ substituents displayed good-to-excellent reactivity. Notably, the technology is amenable to cross-couplings of alkyl acid chloride, which are problematic considering their higher reactivity toward hydrolysis or ketene formation under basic aqueous conditions $(19,20)$. For example, lauroyl chloride displayed good-toexcellent reactivity with various arylboronic acids, including terminal vinyl (20) and thiophenyl (19) groups. Other sulfurcontaining heterocycles also exhibit excellent reactivity under these mild catalytic conditions $(9,10,12-14,19,21,22,24$, 27, 30). However, pyridyl-containing substrates completely inhibited the reaction, which may be due to the disruption of Pd-amphiphile binding due to competitive Pd-pyridine binding (for details see page S25 in the Supporting Information).

To evaluate the stability of acid chloride $\mathbf{1}$, three parallel reactions were conducted by dispersing benzoyl chloride in the basic micellar solution using standard reaction conditions as illustrated in Table 3. A nitrogen nucleophile morpholine was introduced to individual reactions at 0,30 , and $60 \mathrm{~min}$, respectively. The amount of amide formation (1a) was monitored by GC-MS analysis. The \% conversions to 1a 
Table 2. Substrate Scope for Ketone Synthesis ${ }^{a}$

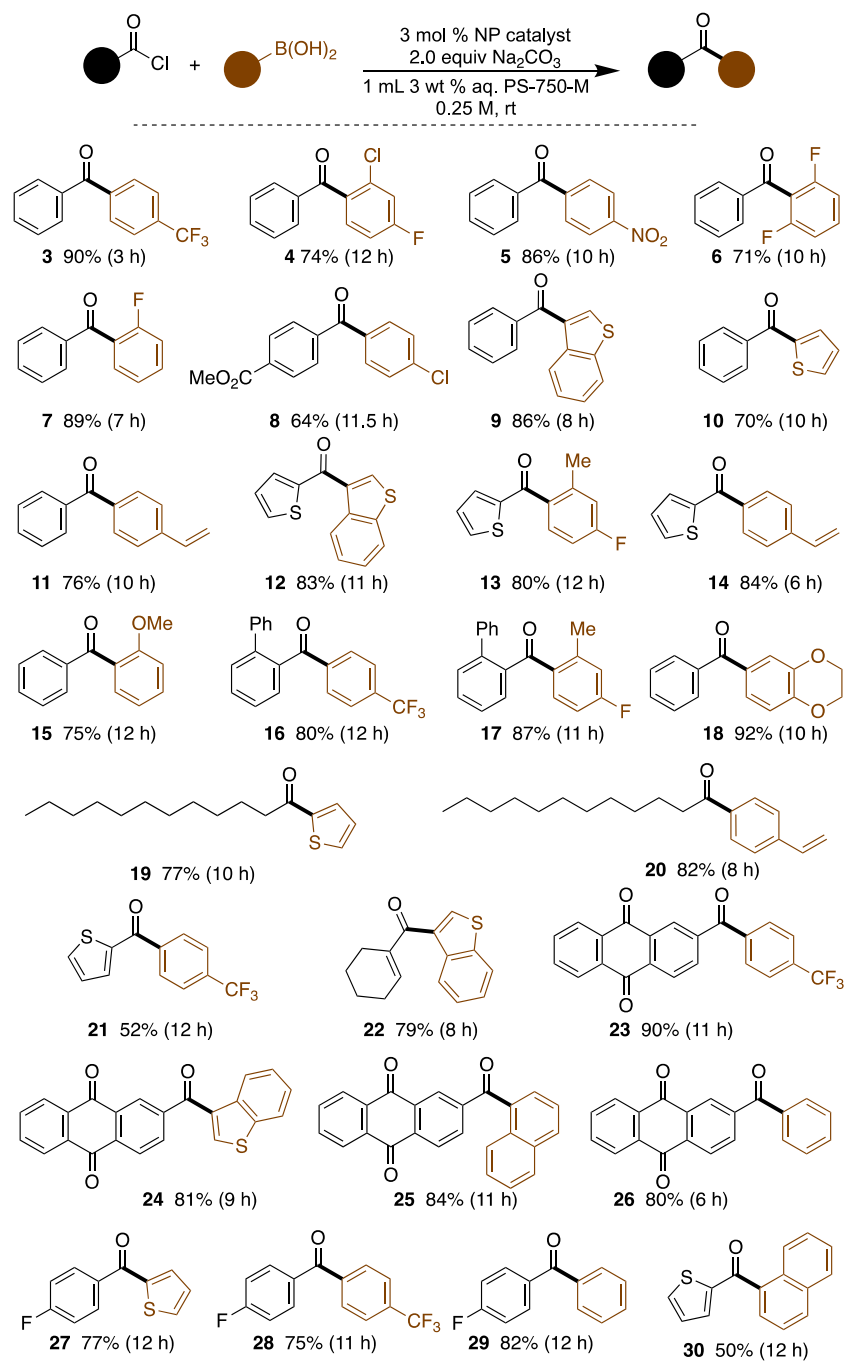

${ }^{a}$ Conditions: acid chloride $(0.25 \mathrm{mmol})$, arylboronic acid $(0.275$ mmol), NP catalyst $(3 \mathrm{~mol} \%), \mathrm{Na}_{2} \mathrm{CO}_{3}(0.5 \mathrm{mmol}), 1 \mathrm{~mL}$ of $3 \mathrm{wt} \%$ aq. PS-750-M, $23{ }^{\circ} \mathrm{C}$; all yields are isolated.

Table 3. Stability Analysis of Acid Chloride ${ }^{a}$

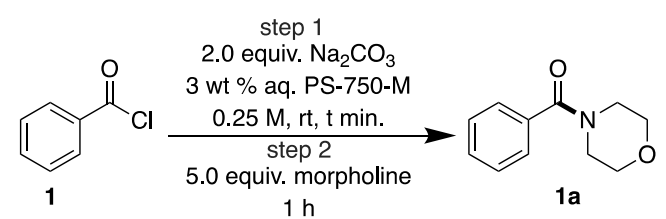

$\begin{array}{ccc}\text { entry } & \text { time }(\min ) & \text { 1a (GC-MS conversion) }(\%) \\ 1 & 0 & 89 \\ 2 & 30 & 63 \\ 3 & 60 & 48\end{array}$

${ }^{a}$ Conditions: Step $1,1(0.5 \mathrm{mmol}), \mathrm{Na}_{2} \mathrm{CO}_{3}(1.0 \mathrm{mmol}), 2 \mathrm{~mL} 3 \mathrm{wt}$ $\%$ aq. PS-750-M, $23{ }^{\circ} \mathrm{C}$, $t$ min.; step 2 , morpholine (2.5 mmol), 0-23 ${ }^{\circ} \mathrm{C}, 1 \mathrm{~h}$; conversions based on GC-MS using mesitylene $(0.5 \mathrm{mmol})$ as an internal standard.

provide an indirect idea of stability of acid chloride under these conditions (Table 3, entries 1-3; for details, see page S24 in the Supporting Information). On the basis of conversion to 1a, approximately half of $\mathbf{1}$ was hydrolyzed but the remaining still existed after $1 \mathrm{~h}$. This indicates that the boronic acid may further play a role in determining the stability of acid chlorides by lowering the overall $\mathrm{pH}$ of the reaction mixture. Therefore, we determined the $\mathrm{pH}$ of a standard reaction mixture before and after the addition of boronic acid 2. The reaction mixture's $\mathrm{pH}$ changed from 9.33 to 8.76 . (for details, see page S23 in the Supporting Information). On the basis of these results, both the aqueous nanomicelles and arylbornic acid possibly play a significant role in determining the stability of acid chloride in aqueous medium.

Next, the activity of these NPs were evaluated for the ligandfree Suzuki-Miyaura (SM) couplings under mild micellar conditions. Considering this reaction's broad importance, ${ }^{5-60}$ the ability to carry out this transformation without using any phosphine ligands under micellar conditions would be of great significance. Similar to the NP activity toward cross-couplings of acid chlorides, good-to-excellent catalytic activity was observed for SM couplings of functionalized aryl and heteroaryl halides and aryl- and heteroarylboronic acids (Table 4, 31-50). Excellent functional group tolerance was

Table 4. Substrate Scope for Suzuki-Miyaura Coupling ${ }^{a}$

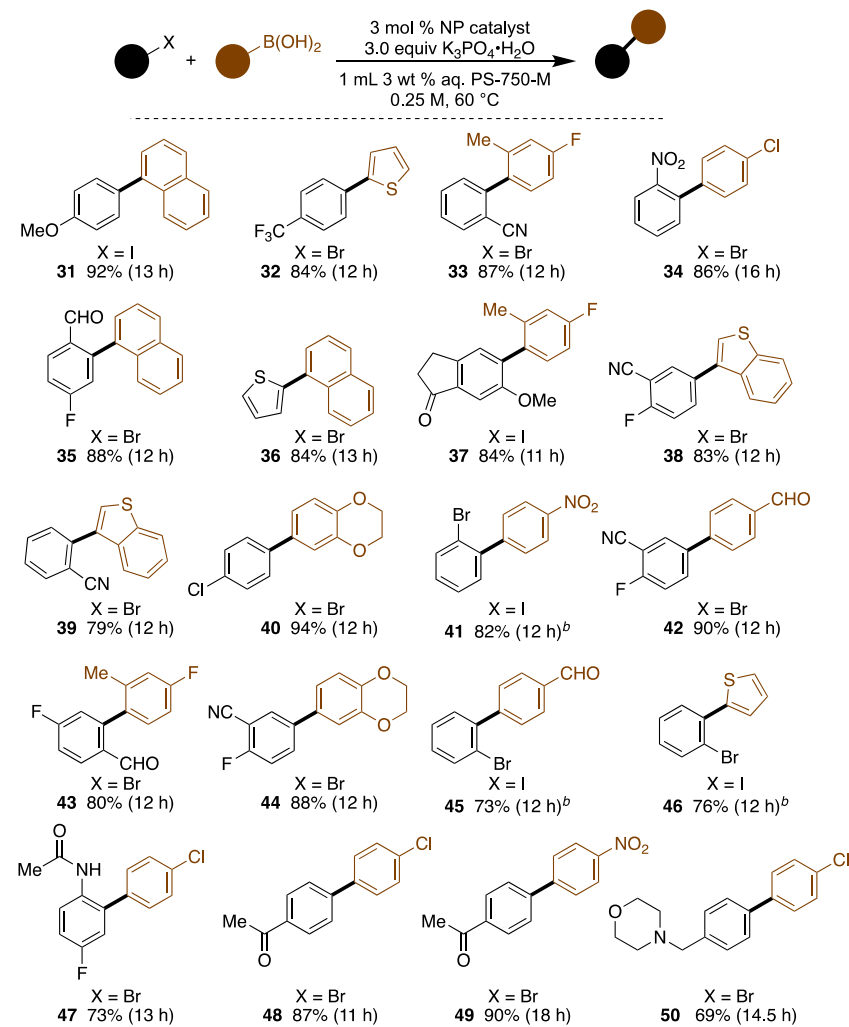

${ }^{a}$ Conditions: aryl halide $(0.25 \mathrm{mmol})$, arylboronic acid $(0.275$ $\mathrm{mmol}), \mathrm{NP}$ catalyst $(3 \mathrm{~mol} \%), \mathrm{K}_{3} \mathrm{PO}_{4} \cdot \mathrm{H}_{2} \mathrm{O}(0.75 \mathrm{mmol}), 1 \mathrm{~mL}$ of 3 wt $\%$ aq. PS-750-M, $60{ }^{\circ} \mathrm{C}$; all yields are isolated. ${ }^{b}$ Reaction temperature is $45{ }^{\circ} \mathrm{C}$ instead of $60{ }^{\circ} \mathrm{C}$.

also observed in this transformation. Functional groups such as formyl $(35,42,43,45)$, chloro $(34,40,47,48,50)$, cyano (33, $38,39,42,44)$, fluoro $(33,35,37,38,42-44,47)$, nitro (34, $41,49)$, ketone $(37,48,49)$, trifluoromethyl (32), and amide (47) were all well-tolerated. Various heterocyclic boronic acids possessing benzodioxane $(40,44)$, benzothiophene $(38,39)$, and thiophene $(32,46)$ residues showed good-to-excellent reactivity. Notably, substrates susceptible to $\alpha$-arylation were well tolerated, and no such side-product was observed $(37,48$, 
49). Even though these NPs are compatible for the coupling of broad range of electrophiles and nucleophiles, these NPs are non-recyclable in nature.

To demonstrate the scalability of these transformations, we performed several reactions on a preparative scale. For example, benzoylation of 4-trifluoromethyl phenylboronic acid 2 was performed at the $20 \mathrm{mmol}$ scale to obtain the desired ketone 3 in $86 \%$ isolated yield (Scheme 2A; see pages

\section{Scheme 2. Scalability Test}

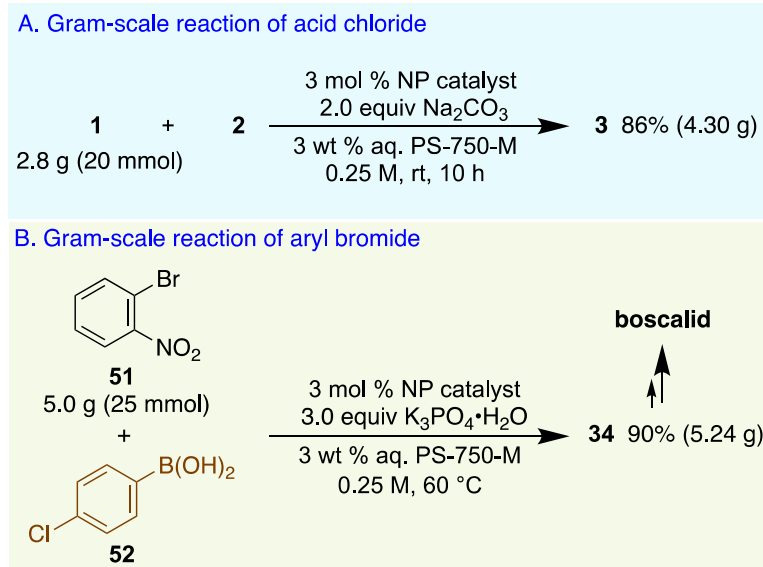

S17 and 18 in the Supporting Information). Likewise, these $\operatorname{Pd}(0)$ NPs were also employed on multigram scale $(25 \mathrm{mmol})$ synthesis of intermediate 34 , a boscalid synthon (Scheme 2B). The coupling of 1-bromo-2-nitrobenzene (51) with 4chlorophenyl boronic acid (52) under mild micellar conditions provides 34 in $90 \%$ isolated yield. The yields in these largescale reactions are similar to those obtained in small-scale reactions depicted in Tables 2 and 3.

Further, this technology's application was evaluated on a compound from Merck's informer library (Scheme 3A; for

\section{Scheme 3. One-Pot Suzuki Couplings and Selective Hydrogenation}

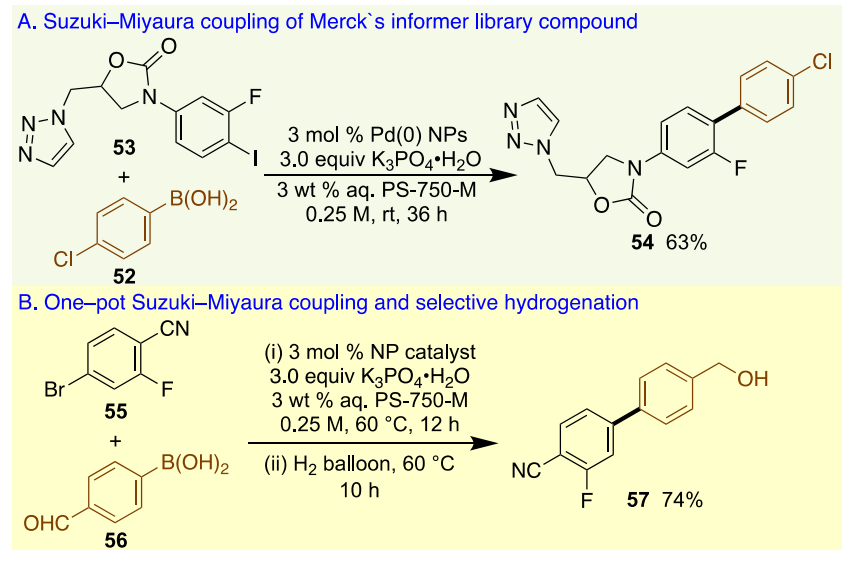

details, see page S19 in the Supporting Information). The Suzuki-Miyaura coupling of a highly functionalized triazolecontaining oxazolidine derivative $\mathbf{5 3}$ with boronic acid $\mathbf{5 2}$ afforded the desired product $\mathbf{5 4}$ in $63 \%$ isolated yield. Another exciting feature of aqueous micellar nanocatalysis is the ability to enable sequential reactions without isolation of intermediates. Hydrogenation reactions under micellar conditions are often selective and do not demand high hydrogen pressure because of the enhanced solubility of hydrogen in the nanomicelles. ${ }^{25}$ Anticipating that our $\operatorname{Pd}(0)$ NPs would also perform selective hydrogenation, we extended our nanocatalysis scope to one-pot reactions involving cross-coupling and selective carbonyl reduction (Scheme 3B; see page S20 in the Supporting Information). After the SM coupling of 5bromo-2-fluorobenzonitrile (55) with 4-formyl phenylboronic acid (56), the aldehyde group of the resultant biaryl 42 was selectively reduced in the presence of the sensitive fluoro and cyano groups to the corresponding benzyl alcohol 57 with excellent isolated yield (74\%). Notably, the same NPs sequentially enabled both transformations in a single pot, simply by introducing hydrogen.

\section{CONCLUSION}

In conclusion, by harnessing the synergy between $\operatorname{Pd}(0)$ and PS-750-M, phosphine ligand-free Pd(0) NPs are developed for sustainable Suzuki-Miyaura couplings of acid chlorides and aryl halides. The synthetic protocols for NP formation and subsequent catalytic reactions are green and do not require organic solvents or harsh reductants. In these NPs, PS-750-M plays a vital role in stabilizing the $\operatorname{Pd}(0)$, circumventing the need for phosphine ligands. The NPs are active for high-valued transformations with broad substrate scope and excellent functional group tolerance. These NPs provide a convenient and scalable new nanotechnology that is benign by design, ecofriendly, and affordable.

\section{ASSOCIATED CONTENT}

\section{Supporting Information}

The Supporting Information is available free of charge at https://pubs.acs.org/doi/10.1021/jacsau.1c00236.

Materials and methods, supplementary figures, supplementary tables, supplementary schemes, and analytical data (PDF)

\section{AUTHOR INFORMATION}

\section{Corresponding Authors}

Sachin Handa - Department of Chemistry, University of Louisville, Louisville, Kentucky 40292, United States; ○ orcid.org/0000-0002-7635-5794; Email: sachin.handa@ louisville.edu

David K. Leahy - Process Chemistry Development, Takeda Pharmaceuticals International, Cambridge, Massachusetts 02139, United States; Present Address: Biohaven Pharmaceuticals, 215 Church Street, New Haven, CT, 06510; 다 orcid.org/0000-0003-4128-7792; Email: david.leahy@takeda.com

\section{Authors}

Tharique N. Ansari - Department of Chemistry, University of Louisville, Louisville, Kentucky 40292, United States

Sudripet Sharma - Department of Chemistry, University of Louisville, Louisville, Kentucky 40292, United States

Susanta Hazra - Department of Chemistry, University of Louisville, Louisville, Kentucky 40292, United States

Jacek B. Jasinski - Materials Characterization, Conn Center for Renewable Energy Research, University of Louisville, Louisville, Kentucky 40292, United States; 이이.org/ 0000-0002-1297-6145 
Andrew J. Wilson - Department of Chemistry, University of Louisville, Louisville, Kentucky 40292, United States; (ㄱ) orcid.org/0000-0003-3427-810X

Frederick Hicks - Process Chemistry Development, Takeda Pharmaceuticals International, Cambridge, Massachusetts 02139, United States

Complete contact information is available at: https://pubs.acs.org/10.1021/jacsau.1c00236

\section{Author Contributions}

The manuscript was written through contributions of all authors. All authors have given approval to the final version of the manuscript.

Notes

The authors declare no competing financial interest.

\section{ACKNOWLEDGMENTS}

This research was supported in part by the National Science Foundation under award number CHE-2044778. We also warmly acknowledge the partial financial support from Takeda Pharmaceuticals. We sincerely thank Spencer Dreher and Guy Humphrey (Merck) for donation of the aryl halides from their Merck informer library.

\section{REFERENCES}

(1) Johansson Seechurn, C. C. C.; Kitching, M. O.; Colacot, T. J.; Snieckus, V. Palladium-Catalyzed Cross-Coupling: A Historical Contextual Perspective to the 2010 Nobel Prize. Angew. Chem., Int. Ed. 2012, 51, 5062-5085.

(2) Nicolaou, K. C.; Bulger, P. G.; Sarlah, D. Palladium-Catalyzed Cross-Coupling Reactions in Total Synthesis. Angew. Chem., Int. Ed. 2005, 44, 4442-4489.

(3) Li, H.; Johansson Seechurn, C. C. C.; Colacot, T. J. Development of Preformed Pd Catalysts for Cross-Coupling Reactions, beyond the 2010 Nobel Prize. ACS Catal. 2012, 2, 1147-1164.

(4) Gardner, B. M.; Seechurn, C. C. C. J.; Colacot, T. J. Industrial Milestones in Organometallic Chemistry. Organometallic Chemistry in Industry. 2020, 1-22.

(5) Colacot, T. J. C. C. C. J. S. Organometallic Chemistry in Industry: A Practical Approach. In Focus on Catalysts; Colacot, T., Ed.; Wiley, 2020; Vol. 2020, pp 336.

(6) Buchwald, S. L.; Mauger, C.; Mignani, G.; Scholz, U. IndustrialScale Palladium-Catalyzed Coupling of Aryl Halides and Amines - A Personal Account. Adv. Synth. Catal. 2006, 348, 23-39.

(7) Suzuki, A. Cross-Coupling Reactions Of Organoboranes: An Easy Way To Construct C-C Bonds (Nobel Lecture). Angew. Chem., Int. Ed. 2011, 50, 6722-6737.

(8) Negishi, E. Magical Power of Transition Metals: Past, Present, and Future (Nobel Lecture). Angew. Chem., Int. Ed. 2011, 50, 67386764.

(9) Beletskaya, I. P.; Cheprakov, A. V. The Heck Reaction as a Sharpening Stone of Palladium Catalysis. Chem. Rev. 2000, 100, 3009-3066.

(10) Hartwig, J. F. Carbon-Heteroatom Bond Formation Catalysed by Organometallic Complexes. Nature 2008, 455, 314-322.

(11) Kalyani, D.; Deprez, N. R.; Desai, L. V.; Sanford, M. S. Oxidative C-H Activation/C-C Bond Forming Reactions: Synthetic Scope and Mechanistic Insights. J. Am. Chem. Soc. 2005, 127, 73307331.

(12) Neufeldt, S. R.; Sanford, M. S. Controlling Site Selectivity in Palladium-Catalyzed C-H Bond Functionalization. Acc. Chem. Res. 2012, 45, 936-946.
(13) Ackermann, L. Carboxylate-Assisted Transition-Metal-Catalyzed C-H Bond Functionalizations: Mechanism and Scope. Chem. Rev. 2011, 111, 1315-1345.

(14) Ruiz-Castillo, P.; Buchwald, S. L. Applications of PalladiumCatalyzed C-N Cross-Coupling Reactions. Chem. Rev. 2016, 116, 12564-12649.

(15) Rayadurgam, J.; Sana, S.; Sasikumar, M.; Gu, Q. Palladium Catalyzed C-C and C-N Bond Forming Reactions: An Update on the Synthesis of Pharmaceuticals from 2015-2020. Org. Chem. Front. 2021, 8, 384-414.

(16) Hartwig, J. F. Evolution of a Fourth Generation Catalyst for the Amination and Thioetherification of Aryl Halides. Acc. Chem. Res. 2008, 41, 1534-1544.

(17) Deangelis, A. J.; Gildner, P. G.; Chow, R.; Colacot, T. J. Generating Active L-Pd(0) via Neutral or Cationic $\pi$-Allylpalladium Complexes Featuring Biaryl/Bipyrazolylphosphines: Synthetic, Mechanistic, and Structure-Activity Studies in Challenging Cross-Coupling Reactions. J. Org. Chem. 2015, 80, 6794-6813.

(18) Surry, D. S.; Buchwald, S. L. Dialkylbiaryl Phosphines in PdCatalyzed Amination: A User's Guide. Chem. Sci. 2011, 2, 27-50.

(19) Favier, I.; Pla, D.; Gómez, M. Palladium Nanoparticles in Polyols: Synthesis, Catalytic Couplings, and Hydrogenations. Chem. Rev. 2020, 120, 1146-1183.

(20) Handa, S.; Wang, Y.; Gallou, F.; Lipshutz, B. H. Sustainable FePpm Pd Nanoparticle Catalysis of Suzuki-Miyaura Cross-Couplings in Water. Science 2015, 349 (6252), 1087-1091.

(21) Handa, S.; Jin, B.; Bora, P. P.; Wang, Y.; Zhang, X.; Gallou, F.; Reilly, J.; Lipshutz, B. H. Sonogashira Couplings Catalyzed by Fe Nanoparticles Containing ppm Levels of Reusable Pd, under Mild Aqueous Micellar Conditions. ACS Catal. 2019, 9, 2423-2431.

(22) Cortes-Clerget, M.; Akporji, N.; Takale, B. S.; Wood, A.; Landstrom, E.; Lipshutz, B. H. Earth-Abundant and Precious Metal Nanoparticle Catalysis. In Nanoparticles in Catalysis; Kobayashi, S., Ed.; Topics in Organometallic Chemistry; Springer, 2020; Vol. 66, pp 77-129. DOI: $10.1007 / 3418 \quad 202036$

(23) Farina, V. High-Turnover Palladium Catalysts in CrossCoupling and Heck Chemistry: A Critical Overview. Adv. Synth. Catal. 2004, 346, 1553-1582.

(24) Hamasaka, G.; Roy, D.; Tazawa, A.; Uozumi, Y. Arylation of Terminal Alkynes by Aryl Iodides Catalyzed by a Parts-per-Million Loading of Palladium Acetate. ACS Catal. 2019, 9, 11640-11646.

(25) Bihani, M.; Bora, P. P.; Nachtegaal, M.; Jasinski, J. B.; Plummer, S.; Gallou, F.; Handa, S. Microballs Containing $\mathrm{Ni}(0) \operatorname{Pd}(0)$ Nanoparticles for Highly Selective Micellar Catalysis in Water. ACS Catal. 2019, 9, 7520-7526.

(26) Somorjai, G. A.; Li, Y. Selective Nanocatalysis of Organic Transformation by Metals: Concepts, Model Systems, and Instruments. Top. Catal. 2010, 53, 832-847.

(27) Ansari, T. N.; Taussat, A.; Clark, A. H.; Nachtegaal, M.; Plummer, S.; Gallou, F.; Handa, S. Insights on Bimetallic Micellar Nanocatalysis for Buchwald-Hartwig Aminations. ACS Catal. 2019, 9, 10389-10397.

(28) Da Silva, F. P.; Fiorio, J. L.; Rossi, L. M. Tuning the Catalytic Activity and Selectivity of Pd Nanoparticles Using Ligand-Modified Supports and Surfaces. ACS Omega 2017, 2, 6014-6022.

(29) Chen, J.; Zhang, Q.; Wang, Y.; Wan, H. Size-Dependent Catalytic Activity of Supported Palladium Nanoparticles for Aerobic Oxidation of Alcohols. Adv. Synth. Catal. 2008, 350, 453-464.

(30) Özkar, S.; Finke, R. G. Palladium(0) Nanoparticle Formation, Stabilization, and Mechanistic Studies: $\mathrm{Pd}(\mathrm{acac})_{2}$ as a Preferred Precursor, $\left[\mathrm{Bu}_{4} \mathrm{~N}\right]_{2} \mathrm{HPO}_{4}$ Stabilizer, plus the Stoichiometry, Kinetics, and Minimal, Four-Step Mechanism of the Palladium Nanoparticle Formation and Subsequ. Langmuir 2016, 32, 3699-3716.

(31) Handa, S.; Andersson, M. P.; Gallou, F.; Reilly, J.; Lipshutz, B. H. HandaPhos: A General Ligand Enabling Sustainable Ppm Levels of Palladium-Catalyzed Cross-Couplings in Water at Room Temperature. Angew. Chem., Int. Ed. 2016, 55, 4914-4918.

(32) Doherty, S.; Knight, J. G.; Backhouse, T.; Abood, E.; Al-Shaikh, H.; Clemmet, A. R.; Ellison, J. R.; Bourne, R. A.; Chamberlain, T. W.; 
Stones, R.; et al. Heteroatom Donor-Decorated Polymer-Immobilized Ionic Liquid Stabilized Palladium Nanoparticles: Efficient Catalysts for Room-Temperature Suzuki-Miyaura Cross-Coupling in Aqueous Media. Adv. Synth. Catal. 2018, 360, 3716-3731.

(33) Liu, J.; Ruffini, N.; Pollet, P.; Llopis-Mestre, V.; Dilek, C.; Eckert, C. A.; Liotta, C. L.; Roberts, C. B. More Benign Synthesis of Palladium Nanoparticles in Dimethyl Sulfoxide and Their Extraction into an Organic Phase. Ind. Eng. Chem. Res. 2010, 49, 8174-8179.

(34) Nagata, T.; Obora, Y. N, N-Dimethylformamide-Protected Single-Sized Metal Nanoparticles and Their Use as Catalysts for Organic Transformations. ACS Omega 2020, 5, 98-103.

(35) Ishida, J.; Nakatsuji, M.; Nagata, T.; Kawasaki, H.; Suzuki, T.; Obora, Y. Synthesis and Characterization of N, N-Dimethylformamide-Protected Palladium Nanoparticles and Their Use in the SuzukiMiyaura Cross-Coupling Reaction. ACS Omega 2020, 5, 9598-9604. (36) Yang, S.; Dong, J.; Yao, Z.; Shen, C.; Shi, X.; Tian, Y.; Lin, S.; Zhang, X. One-Pot Synthesis of Graphene-Supported Monodisperse Pd Nanoparticles as Catalyst for Formic Acid Electro-Oxidation. Sci. Rep. 2015, 4, 1-6.

(37) Lipshutz, B. H.; Gallou, F.; Handa, S. Evolution of Solvents in Organic Chemistry. ACS Sustainable Chem. Eng. 2016, 4, 5838-5849.

(38) Gallou, F.; Lipshutz, B. H. Organometallic Processes in Water. In Topics in Organometallic Chemistry; Springer: Berlin, 2018; pp 118. DOI: $10.1007 / 3418 \quad 2018 \quad 17$

(39) Ansari, T. N.; Handa, S.; Gallou, F. Cross-Couplings in Water A Better Way to Assemble New Bonds. In Organometallic Chemistry in Industry: A Practical Approach, 1st ed.; Colacot, T. J.; Seechurn, C. C. C. J., Eds.; Wiley, 2020; pp 203-235.

(40) Brals, J.; Smith, J. D.; Ibrahim, F.; Gallou, F.; Handa, S. MicelleEnabled Palladium Catalysis for Convenient $\mathrm{sp}^{2}-\mathrm{sp}^{3}$ Coupling of Nitroalkanes with Aryl Bromides in Water Under Mild Conditions. ACS Catal. 2017, 7, 7245-7250.

(41) Smith, J. D.; Ansari, T. N.; Andersson, M. P.; Yadagiri, D.; Ibrahim, F.; Liang, S.; Hammond, G. B.; Gallou, F.; Handa, S. Micelle-Enabled Clean and Selective Sulfonylation of Polyfluoroarenes in Water under Mild Conditions. Green Chem. 2018, 20, 17841790.

(42) Sharma, S.; Buchbinder, N. W.; Braje, W. M.; Handa, S. Fast Amide Couplings in Water: Extraction, Column Chromatography, and Crystallization Not Required. Org. Lett. 2020, 22, 5737-5740.

(43) Bora, P. P.; Bihani, M.; Plummer, S.; Gallou, F.; Handa, S. Shielding Effect of Micelle for Highly Effective and Selective Monofluorination of Indoles in Water. ChemSusChem 2019, 12, 3037-3042.

(44) Duong, U.; Ansari, T. N.; Parmar, S.; Sharma, S.; Kozlowski, P. M.; Jasinski, J. B.; Plummer, S.; Gallou, F.; Handa, S. Nanochannels in Photoactive Polymeric $\mathrm{Cu}(\mathrm{I})$ Compatible for Efficient Micellar Catalysis: Sustainable Aerobic Oxidations of Alcohols in Water. ACS Sustainable Chem. Eng. 2021, 9, 2854-2860.

(45) Handa, S.; Ibrahim, F.; Ansari, T. N.; Gallou, F. $\pi$ Allylpalladium Species in Micelles of FI-750-M for Sustainable and General Suzuki-Miyaura Couplings of Unactivated Quinoline Systems in Water. ChemCatChem 2018, 10, 4229-4233.

(46) Bihani, M.; Ansari, T. N.; Finck, L.; Bora, P. P.; Jasinski, J. B.; Pavuluri, B.; Leahy, D. K.; Handa, S. Scalable $\alpha$-Arylation of Nitriles in Aqueous Micelles Using Ultrasmall Pd Nanoparticles: Surprising Formation of Carbanions in Water. ACS Catal. 2020, 10, 6816-6821.

(47) Duong, U. T.; Gade, A. B.; Plummer, S.; Gallou, F.; Handa, S. Reactivity of Carbenes in Aqueous Nanomicelles Containing Palladium Nanoparticles. ACS Catal. 2019, 9, 10963-10970.

(48) Ansari, T. N.; Jasinski, J. B.; Leahy, D. K.; Handa, S. MetalMicelle Cooperativity: Phosphine Ligand-Free Ultrasmall Palladium(II) Nanoparticles for Oxidative Mizoroki-Heck-Type Couplings in Water at Room Temperature. JACS Au 2021, 1, 308-315.

(49) Sawai, K.; Tatumi, R.; Nakahodo, T.; Fujihara, H. Asymmetric Suzuki-Miyaura Coupling Reactions Catalyzed by Chiral Palladium Nanoparticles at Room Temperature. Angew. Chem., Int. Ed. 2008, 47, 6917-6919.
(50) Xin, B.; Zhang, Y.; Cheng, K. Phosphine-Free Cross-Coupling Reaction of Arylboronic Acids with Carboxylic Anhydrides or Acyl Chlorides in Aqueous Media. J. Org. Chem. 2006, 71, 5725-5731.

(51) Blangetti, M.; Rosso, H.; Prandi, C.; Deagostino, A.; Venturello, P. Suzuki-Miyaura Cross-Coupling in Acylation Reactions, Scope and Recent Developments. Molecules 2013, 18, 1188-1213.

(52) Buchspies, J.; Szostak, M. Recent Advances in Acyl Suzuki Cross-Coupling. Catalysts 2019, 9, 53-76.

(53) Klingler, F. D. Asymmetric Hydrogenation of Prochiral Amino Ketones to Amino Alcohols for Pharmaceutical Use. Acc. Chem. Res. 2007, 40, 1367-1376.

(54) Raynbird, M. Y.; Sampson, J. B.; Smith, D. A.; Forsyth, S. M.; Moseley, J. D.; Wells, A. S. Ketone Reductase Biocatalysis in the Synthesis of Chiral Intermediates Toward Generic Active Pharmaceutical Ingredients. Org. Process Res. Dev. 2020, 24, 1131-1140.

(55) Okitsu, T.; Ogasahara, M.; Wada, A. Convergent Synthesis of Dronedarone, an Antiarrhythmic Agent. Chem. Pharm. Bull. 2016, 64, 1149-1153.

(56) Caille, S.; Cui, S.; Faul, M. M.; Mennen, S. M.; Tedrow, J. S.; Walker, S. D. Molecular Complexity as a Driver for Chemical Process Innovation in the Pharmaceutical Industry. J. Org. Chem. 2019, 84, $4583-4603$.

(57) Caron, S.; Dugger, R. W.; Ruggeri, S. G.; Ragan, J. A.; Ripin, D. H. B. Large-Scale Oxidations in the Pharmaceutical Industry. Chem. Rev. 2006, 106, 2943-2989.

(58) Takale, B. S.; Thakore, R. R.; Irvine, N. M.; Schuitman, A. D.; Li, X.; Lipshutz, B. H. Sustainable and Cost-Effective Suzuki-Miyaura Couplings toward the Key Biaryl Subunits of Arylex and Rinskor Active. Org. Lett. 2020, 22, 4823-4827.

(59) Gallou, F.; Isley, N. A.; Ganic, A.; Onken, U.; Parmentier, M. Surfactant Technology Applied toward an Active Pharmaceutical Ingredient: More than a Simple Green Chemistry Advance. Green Chem. 2016, 18, 14-19.

(60) Parmentier, M.; Wagner, M.; Wickendick, R.; Baenziger, M.; Langlois, A.; Gallou, F. A General Kilogram Scale Protocol for SuzukiMiyaura Cross-Coupling in Water with TPGS-750-M Surfactant. Org. Process Res. Dev. 2020, 24, 1536-1542. 